\title{
STABILIZATION PHENOMENA IN KAC-MOODY ALGEBRAS AND QUIVER VARIETIES
}

\author{
BEN WEBSTER
}

\begin{abstract}
Let $X$ be the Dynkin diagram of a symmetrizable Kac-Moody algebra, and $X_{0}$ a subgraph with all vertices of degree 1 or 2 . Using the crystal structure on the components of quiver varieties for $X$, we show that if we expand $X$ by extending $X_{0}$, the branching multiplicities and tensor product multiplicities stabilize, provided the weights involved satisfy a condition which we call "depth" and are supported outside $X_{0}$. This extends a theorem of Kleber and Viswanath.

Furthermore, we show that the weight multiplicities of such representations are polynomial in the length of $X_{0}$, generalizing the same result for $A_{\ell}$ by Benkart, et al.
\end{abstract}

\section{INTRODUCTION}

1.1. Background. If $X=(\mathcal{V}, \mathcal{E})$ is the Dynkin diagram of a symmetrizable Kac-Moody algebra, then we can construct a series of diagrams $X(m)$ by attaching an $A_{m}$ "tail" to a fixed vertex. For large $m$, one can consider weights supported on the "head" ( $X$ and the adjacent portion of the tail) and on the end of the tail, and vanishing on a large section of tail in between. We can extend such a weight to $X(m)$ for all larger values of $m$ by simply allowing this gap to grow. We might hope that there would be some interesting asymptotic behavior as $m$ goes to infinity.

In the case where $X=A_{m}$, this construction was first studied by Hanlon in Han85, and R. Brylinski in Bry89. They discovered a number of interesting stabilization phenomena, most of which seem specific to the $A_{m}$ case. However, in KV04, Vis05, Kleber and Viswanath show that one aspect does carry over:

Theorem 1.1. (Kleber, Viswanath) Let $\lambda, \mu, \nu$ be a deep triple of weights (as defined in Section 圆) on $X(m)$ supported on the head and

2000 Mathematics Subject Classification. 17B67.

This material is based upon work supported under a National Science Foundation Graduate Research Fellowship and partially supported by the RTG grant DMS0354321. 
end of tail as described above. Then the tensor product multiplicity $c_{\mu, \nu}^{\lambda}(m)$ for the Kac-Moody algebra $\mathfrak{g}(m)=\mathfrak{g}(X(m))$ stabilizes for $m$ sufficiently large.

The proof is based on an analysis of the crystal graph of a representation of $\mathfrak{g}(m)$ via Littlemann paths and the following easy consequence of the theory of crystal graphs due to Kashiwara and others (see, for example, [CP95]

Theorem 1.2. Let $B_{\mu}$ be the crystal (graph) corresponding to the representation $V_{\mu}$. Then

$$
c_{\mu \nu}^{\lambda}=\#\left\{x \in B_{\mu} \mid \operatorname{wt}(x)=\lambda-\nu, \tilde{e}_{i}^{\nu\left(\alpha_{i}^{\vee}\right)+1} x=0\right\},
$$

where $\tilde{e}_{i}: B_{\mu} \cup\{0\} \rightarrow B_{\mu} \cup\{0\}$ denotes the ith Kashiwara operator.

Our aim in this paper is to generalize Theorem 1.1 by using a variant of Kleber and Viswanath's techniques. We will replace Littelmann paths with the crystal structure on the components of quiver varieties, described in KS97, Sav04.

This approach allows us to extend Theorem 1.1 to a more general class of arrangements. Our results imply the stabilization theorems of KV04, Theorem 2.6] and [Vis05, Theorem 2.5], though some care is required to see this in the case of [KV04]: the restriction of "extensibility" there exactly implies that tensor product multiplicities vanish in the non-deep case. Kleber and Viswanath refer to this as the "number-ofboxes condition" by analogy with $\mathfrak{g l}_{n}$, where it implies that the number of boxes in the Young diagram adds under tensor product.

1.2. Summary of results. Let $C$ be a generalized Cartan matrix, in the sense of Kac, and let $C_{0}$ be a subset of the row/column set (identified by transpose) such that each corresponding row or column contains at most 2 non-zero off-diagonal entries, and all such entries are -1 . For simplicity, we also require that no minor consisting of rows from $C_{0}$ is the Cartan matrix of $\tilde{A}_{n}$.

More geometrically, consider the directed graph $X$ with adjacency matrix $A=2 I-C$. Throughout our paper, we will assume that $X$ is the graph corresponding to a symmetrizable Cartan matrix. Then the subgraph $X_{0}$ corresponding to $C_{0}$ must be a union of components

$$
X_{0}=\bigsqcup_{i=1}^{k} X_{i}
$$

with $X_{i} \cong A_{\ell_{i}}$ for some integers $\ell_{1}, \ldots, \ell_{k}$. We call such a subgraph elastic. The symbol $X_{0}$ will denote an elastic subgroup throughout this paper. 
We let $X\left(m_{1}, \ldots, m_{k}\right)=X(\mathbf{m})$ be $X$ with $X_{0}$ replaced by

$$
X_{0}(\mathbf{m})=\bigsqcup_{i=1}^{k} X_{i}\left(m_{i}\right) \cong A_{m_{i}+\ell_{i}} .
$$

That is, we let the selected string in our Dynkin diagram grow by $m_{i}$ edges. This is illustrated in Figure 1.
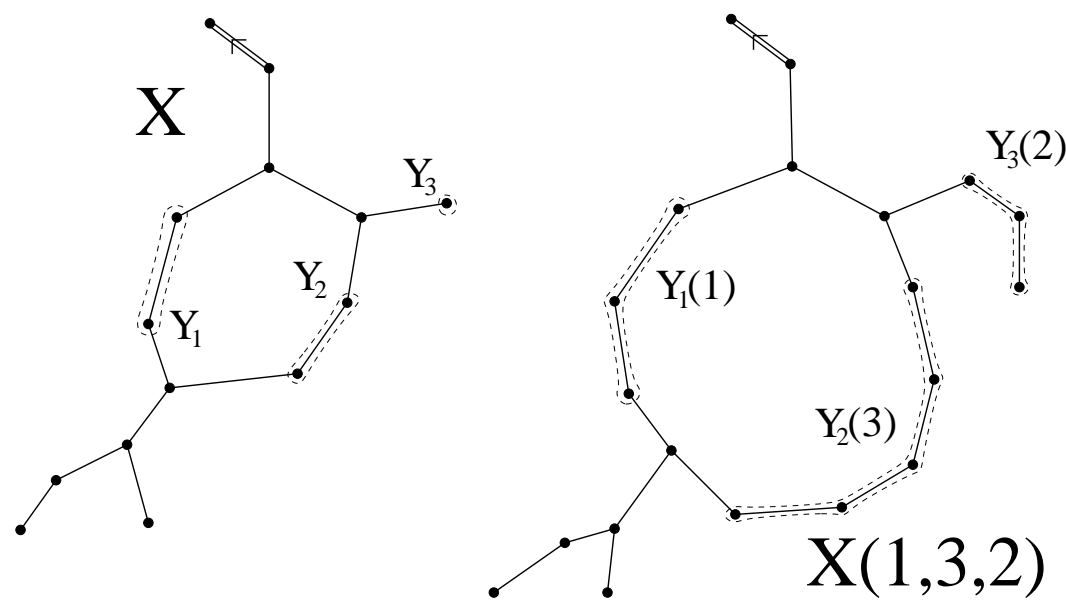

Figure 1. The effect of expansion on a Dynkin diagram

Let $R^{\vee}\left(X_{0}\right)^{\perp}$ be the annihilator of the coroot lattice of $X_{0}$, that is,

$$
R^{\vee}\left(X_{0}\right)^{\perp}=\left\{\delta \in P(X) \mid \delta\left(\alpha_{i}^{\vee}\right)=0, \forall i \in X_{0}\right\}
$$

If $\mu \in R^{\vee}\left(X_{0}\right)^{\perp}$, we will often say it "vanishes on $X_{0}$."

If we have a weight $\mu$ vanishing on $X_{0}$, we can view $\mu$ as a weight of $X(m)$ for all $m$ (there are minor difficulties if the Cartan matrix of $X$ is degenerate, but this will be dealt with in later sections), since $X-X_{0} \cong X(\mathbf{m})-X_{0}(\mathbf{m})$. Fix weights $\mu, \nu, \lambda \in R^{\vee}\left(X_{0}\right)^{\perp}$ and let $\theta=\lambda-\nu$. We let $V(\mu, \mathbf{m})$ denote the corresponding representation with highest weight $\mu$ of the Kac-Moody algebra $\mathfrak{g}(\mathbf{m})$ with Dynkin diagram $X(\mathbf{m})$, and $V(\mu, \mathbf{m})_{\theta}$ its $\theta$-weight space. Furthermore, we let $\mathfrak{t}(\mathbf{m})$ denote the Cartan subalgebra of $\mathfrak{g}(\mathbf{m})$ and $\mathfrak{g}_{0}(\mathbf{m})$ the root subalgebra corresponding to the subdiagram $X_{0}$. Note that $\mathfrak{g}_{\mathfrak{o}}+\mathfrak{t}(\mathbf{m})$ is a finite dimensional reductive algebra with the same Cartan subalgebra as $\mathfrak{g}(\mathbf{m})$. Thus $\mu$ also has an associated irreducible highest weight representation of $\mathfrak{g}_{\mathrm{o}}+\mathfrak{t}(\mathbf{m})$, which we denote $V_{0}(\mu, \mathbf{m})$.

Let us consider the asymptotic behavior of three of the most commonly studied numerical invariants of representations of Kac-Moody algebras: 
- the branching multiplicities fro $\mathrm{m} \mathfrak{g}(\mathbf{m})$ to $\mathfrak{g}_{0}+\mathfrak{t}(\mathbf{m})$ :

$$
b_{\theta}^{\mu}(\mathbf{m})=\operatorname{dim} \operatorname{Hom}_{\mathfrak{g}_{\mathfrak{o}}+\mathfrak{t}(\mathbf{m})}\left(V_{0}(\theta, \mathbf{m}), V(\mu, \mathbf{m})\right)
$$

This is closely connected to the more often-studied branching rule for $\mathfrak{g}_{0}(\mathbf{m})$, but also includes the action of the full torus. This more detailed information will be important later.

- the tensor product multiplicities for $\mathfrak{g}(\mathbf{m})$ representations:

$$
c_{\mu, \nu}^{\lambda}(\mathbf{m})=\operatorname{dim} \operatorname{Hom}_{\mathfrak{g}(\mathbf{m})}(V(\lambda, \mathbf{m}), V(\mu, \mathbf{m}) \otimes V(\nu, \mathbf{m}))
$$

- the weight multiplicities

$$
w_{\theta}^{\mu}(\mathbf{m})=\operatorname{dim} V(\mu, \mathbf{m})_{\theta} .
$$

In the large $\mathbf{m}$ limit, there is a subset of triples of weights called deep which behave exactly as we might hope by extrapolating from KV04 and the case of $A_{\ell}$ :

Theorem 1.3. For any elastic subgraph $X_{0} \subset X$ of the Dynkin graph of a symmetrizable Kac-Moody algebra, and for all weights $\lambda, \mu, \nu$, with $\mu+\nu-\lambda=\mu-\theta$ deep of $j$-depth $\sigma_{j}$, and $m_{i}$ sufficiently large for all $i \in\{1, \ldots, k\}$.

(1) the branching multiplicities $b_{\theta}^{\mu}(\mathbf{m})$ and tensor product multiplicities $c_{\mu, \nu}^{\lambda}(\mathbf{m})$ are independent of $\mathbf{m}$.

(2) the weight multiplicities $w_{\mu}^{\lambda}(\mathbf{m})$ are polynomials in the variables $m_{i}$ of multi-degree $\leq\left(\sigma_{1}, \ldots, \sigma_{k}\right)$.

Unfortunately, these multiplicities remain very mysterious objects and quite difficult to compute. It would be particularly nice if a more explicit combinatorial interpretation beyond Littelmann paths or crystal graphs could be found, at least in some special cases. Even if it were not computationally useful, it would represent a small window into strange world of wild Kac-Moody algebras.

We begin with a brief introduction to deep weights in Section 2, followed in Section 3 by a review the relevant facts about quiver varieties and their connections to representation theory which will be necessary for the proof of this theorem. Then we will prove parts (1) and (2) of Theorem 1.3 in Section 4 and Section 5 respectively.

\section{ACKNOWLEDGMENTS}

The author would like to thank Sankaran Viswanath, Joel Kamnitzer, Alistair Savage and Nicolai Reshetikhin for their advice and help. 
We would also like to express our appreciation for the hospitality of the Banff International Research Station and the efforts of the organizers of the conference "Representations of Kac-Moody Algebras and Combinatorics" held there in March 2005, where this research originated.

\section{Deep Weights}

Let $P(X)$ be the weight lattice of $X$, and $R(X) \subset P(X)$ the root lattice, and let $\alpha_{i}$ be the simple roots and $\alpha_{i}^{\vee}$ the simple coroots. Let $\mathbb{Z}^{\mathcal{V}(X)}$ be the set of maps $\mathcal{V}(X) \rightarrow \mathbb{Z}$. There are natural maps

$$
\begin{aligned}
\omega: P(X) & \rightarrow \mathbb{Z}^{\mathcal{V}(X)} & \alpha: R(X) & \rightarrow \mathbb{Z}^{\mathcal{V}(X)} \\
\lambda & \mapsto\left\{i \mapsto \lambda\left(\alpha_{i}^{\vee}\right)\right\} & \sum_{i \in \mathcal{V}(X)} v_{i} \alpha_{i} & \mapsto\left\{i \mapsto v_{i}\right\}
\end{aligned}
$$

The map $\alpha$ is obviously an isomorphism, since the simple roots $\alpha_{i}$ are linearly independent after choice of a realization (in the sense of Kac).

The map $\omega$ is surjective, but not in general injective. Its kernel is naturally identified with the nullspace of the Cartan matrix.

Still, the properties of representations important to us depend only on $\omega(v)$. If we have weights $\mu^{\prime}, \nu^{\prime}, \lambda^{\prime}$ such that

$$
\begin{aligned}
& \mathbf{w}=\omega(\mu)=\omega\left(\mu^{\prime}\right) \\
& \mathbf{y}=\omega(\nu)=\omega\left(\nu^{\prime}\right) \\
& \mathbf{v}=\alpha(\mu+\nu-\lambda)=\alpha\left(\mu^{\prime}+\nu^{\prime}-\lambda^{\prime}\right)
\end{aligned}
$$

then there is a unique isomorphism $B_{\mu} \cong B_{\mu^{\prime}}$ between their crystals (since $V(\mu) \cong V\left(\mu^{\prime}\right)$ as $\mathfrak{n}^{-}$-modules), so Theorem 1.2 implies that $c_{\mu, \nu}^{\lambda}=c_{\mu^{\prime}, \nu^{\prime}}^{\lambda^{\prime}}$. Thus, we can denote this multiplicity $c_{\mathbf{w}, \mathbf{y}}^{\mathbf{v}}$ without any loss of information.

Similarly, by the same crystal isomorphism, we can see that $b_{\theta}^{\mu}=b_{\theta^{\prime}}^{\mu^{\prime}}$, so we can write $b_{\theta}^{\mu}=b_{\mathbf{v}}^{\mathbf{w}}$ where $\mathbf{w}=\omega(\mu)$ and $\mathbf{v}=\alpha(\mu-\theta)$.

Let $Z \subset X$ be any subgraph. We call a function $\mathbf{v} \in \mathbb{Z}^{\mathcal{V}}(X)$ deep for $Z$ if $v_{i}$ is constant on each connected component $Z_{j}$ of $Z$. The value $\sigma_{j}$ of this constant on $Z_{j}$ is called the $Z_{j}$-depth of $\mathbf{v}$. The depth of $\mathbf{v}$ is the maximum of the $Z_{j}$-depths. In the case where $Z=X_{0}$, we typically write $j$-depth for $X_{j}$-depth.

If $\lambda \in R(X)$, then $\lambda$ is called deep if $\alpha(\lambda)$ is deep. A triple of weights, $(\mu, \nu, \lambda)$ is called deep if $\mu+\nu-\lambda$ is deep. Depths and $j$-depths of weights are defined similarly. 


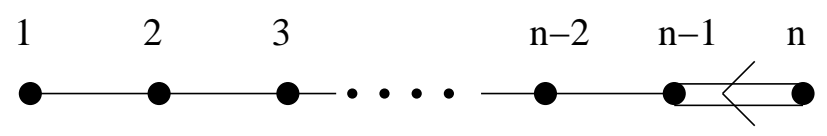

Figure 2. The Dynkin diagram $B_{n}$

2.1. The case of Viswanath. Depth seems like a strong condition, but in fact, non-deep weights have a very hard time staying in the root lattice. In certain cases $\left(A_{n}\right.$, for example), the depth hypothesis in Theorem 1.3 is entirely redundant. Assume $X$ and $X_{0}$ are connected, and $X \backslash X_{0}$ is disconnected, so $X_{0}$ is connecting two separate Dynkin diagrams. This situation has been studied by Viswanath in Vis05]. If these diagrams form an extensible pair as defined in Vis05] then for large $m$, the diagram $X(m)$ has non-degenerate Cartan matrix, and the fundamental weights $\omega_{j}$ make sense. Thus, we have an inclusion $i_{m}: R^{\vee}\left(X_{0}\right)^{\perp} \hookrightarrow R\left(X_{0}(m)\right)^{\perp}$ such that $i_{m}\left(\omega_{j}\right)=\omega_{j^{\prime}}$.

Proposition 2.1. (Viswanath) If $\lambda \in R(X)$ is not deep, then $i_{m}(\lambda) \notin$ $R(X(m))$ for all $m$ sufficiently large. Thus, $c_{\mu, \nu}^{\lambda}(m)$ stabilizes for all weights.

While there are many examples of extensible pairs, there are a lot of cases that are not extensible and Proposition 2.1] fails. For example, while $X=A_{n}$ with $X_{0}$ connected is extensible in this sense, types $B_{n}, C_{n}, D_{n}$ are not, and in these cases counterexamples to Proposition 2.1 are easily located. Interestingly, numerical evidence suggests that even in these cases, tensor product multiplicities may stabilize, but beyond computer computations and the finite and affine cases (as described in [BBL90, BKLS99]), little is known about these cases.

For example, let $X=B_{n}$, with the numbering given in Figure 2.

For all $n, V\left(\omega_{n}\right)$ is the spin representation, and we have the identity

$$
2 \omega_{n}=\sum_{i=1}^{n} i \alpha_{i}
$$

so the labeling of Figure 2 also represents the values of $\alpha\left(2 \omega_{n}\right)$. It follows that $2 \omega_{n} \in R\left(B_{n}\right)$ for all $n$, and is obviously not deep for any $X_{0} \subset X$

Since $V\left(\omega_{n}\right) \cong V\left(\omega_{n}\right)^{*}$, for all $n, c_{\omega_{n}, \omega_{n}}^{0}=1$, even though this triple is not deep. Thus, in this case, and many others, we cannot ignore non-deep weights.

\section{Crystal structures on Quiver varieties}


3.1. Quivers and their representations. Our primary tool will be the geometry of quiver varieties and its connections with representation theory, originally due to Lusztig and Nakajima. See the papers Lus91, Nak94, Nak98, for more details on the construction of quiver varieties and their applications.

A quiver is a directed graph $X=(\mathcal{V}, \mathcal{E})$. A framed representation of a quiver is a choice of:

- two vector spaces, $V_{i}$ and $W_{i}$ for each vertex $i \in \mathcal{V}$,

- maps $x_{e}: V_{\alpha(e)} \rightarrow V_{\omega(e)}$ and $x_{\bar{e}}: V_{\omega(e)} \rightarrow V_{\alpha(e)}$ for each $e \in \mathcal{E}$ (here we use $\alpha(e)$ and $\omega(e)$ to denote the tail and head of the edge $e$ ).

- maps $t_{i}: V_{i} \rightarrow W_{i}$ for each vertex $i \in \mathcal{V}$.

It is often convenient to package together the vector spaces

$$
V=\bigoplus_{i \in \mathcal{V}(X)} V_{i} \quad W=\bigoplus_{i \in \mathcal{V}(X)} W_{i}
$$

and view them as a representation $V \oplus W$ of the framed path algebra $\Pi(X)$. This algebra is generated by elements $x_{P}$ for each path $P$ in $X$, and $j_{i}$ for $i \in \mathcal{V}(X)$, with relations:

$$
\begin{aligned}
x_{P} x_{P^{\prime}} & =x_{P * P^{\prime}}, & j_{i} j_{k} & =0 \text { for all } i, k \in \mathcal{V} \\
x_{P} j_{i} & =0, & j_{i} x_{P} & =0 \text { iff } i \neq \omega(P) .
\end{aligned}
$$

The vector space $V \oplus W$ is naturally a representation of this algebra with $x_{P}$ given by composition of $x_{e}$ for the edges $e \in P$, and the action of $j_{i}$ given by the map $t_{i}$.

Let $\mathcal{I}$ be the ideal generated by all paths of length greater than 0 , and let $\epsilon_{i}(x, t)=\operatorname{dim}(V / \mathcal{I} V)_{i}$ be the dimension of the quotient of the vector space $V_{i}$ by the image of all the paths ending at $i$.

We say a quiver representation

- is nilpotent if for some integer $N, x_{P}=0$ for all paths $P$ with length $\geq N$.

- is stable if there is no subrepresentation contained in $V$.

- satisfies property MM if

$$
\sum_{e \in \mathcal{E}(X)} x_{e} x_{\bar{e}}-x_{\bar{e}} x_{e}=0 .
$$

Definition. A Nakajima quiver representation (or NQR for short) is a stable, nilpotent framed quiver representation which satisfies property MM, along with a system of isomorphisms $\varphi_{i}: W_{i} \rightarrow \mathbb{C}^{w_{i}}$. 
Let $\mathcal{N}(X, V, W)$ be the variety of NQR's for $X$ on the vector space $V \oplus W$.

We let $\Lambda(X, V, W)$ be the quotient of the variety of NQR's on $V \oplus W$ under the obvious action of

$$
G=\prod_{i \in \mathcal{V}} \mathrm{GL}\left(V_{i}\right) \times \mathrm{GL}\left(W_{i}\right) .
$$

The condition of stability implies that $G$ acts freely on $\Lambda(X, V, W)$, so this is a geometric quotient, and using geometric invariant theory, we can give it the structure of a projective variety over $\mathbb{C}$.

This is a Lagrangian subvariety inside the hyperkähler quiver variety defined by Nakajima in Nak94. In fact it, it is the "core," in the sense of Proudfoot Pro, §2.2].

Up to natural isomorphism, this variety only depends on the dimensions

$$
v_{i}=\operatorname{dim} V_{i} \quad w_{i}=\operatorname{dim} W_{i}
$$

Thus, we will usually denote this variety by $\Lambda(X, \mathbf{v}, \mathbf{w})$ without choosing fixed vector spaces of the given dimensions.

3.2. Quiver varieties as moduli spaces. Let $\Lambda(X)$ be the union over all $\mathbf{v}$ and $\mathbf{w}$ of $\Lambda(X, \mathbf{v}, \mathbf{w})$. Then $\Lambda(X)$ also has a moduli theoretic interpretation:

Let $S$ be a scheme over $\mathbb{C}$. Then our notion of a NQR of vector spaces can be extended to an NQR of algebraic vector bundles (i.e. locally free sheaves) over $S$. Following the usual terminology of algebraic geometry, we refer to this as a family of NQR's over $S$. The case of vector spaces is simply when $S=\operatorname{Spec} \mathbb{C}$.

Over $\mathcal{N}(X, V, W)$, there is a natural, $G$-equivariant family $(\tilde{\mathcal{V}} \oplus \tilde{\mathcal{W}}, \tilde{x}, \tilde{t})$ of NQR's, which one may call the tautological NQR. Since $G$ acts freely on $\mathcal{N}(X, V, W)$, this is the pullback of an $\operatorname{NQR}(\mathcal{V} \oplus \mathcal{W}, x, t)$ on $\Lambda(X, \mathbf{v}, \mathbf{w})$, and taking union, on $\Lambda(X)$. We call this the universal NQR.

Consider the cofunctor $\mathcal{N}: \mathrm{Sch} / \mathbb{C} \rightarrow$ Set sending a scheme over $\mathbb{C}$ to the set of isomorphism classes of families of NQR's. As usual, pullback of the universal bundle by $f$, gives a natural transformation of functors $U: \operatorname{Mor}(-, \Lambda(X)) \rightarrow \mathcal{N}$.

Proposition 3.1. The natural transformation $U$ is an isomorphism. That is, the scheme $\Lambda(X)$ represents $\mathcal{N}$.

Proof. Since we can glue morphisms, we can reduce to the case where $S$ is affine and our NQR is on a trivial vector bundle $L_{V} \oplus L_{W}$. $L_{W}$ is already trivialized by the definition of an NQR. Thus, we need only 
pick a basis of nonvanishing sections of $L_{V}$. Taking matrix coefficients, this gives a map from $S$ to $\mathcal{N}\left(X, \oplus \mathbb{C}^{v_{i}}, \oplus \mathbb{C}^{w_{i}}\right)$ and thus by projection to $\Lambda(X, \mathbf{v}, \mathbf{w})$. Obviously, the tautological bundle on $\mathcal{N}\left(X, \oplus \mathbb{C}^{v_{i}}, \oplus \mathbb{C}^{w_{i}}\right)$ pulls back to $L_{V} \oplus L_{W}$. By transitivity of pullback, the universal bundle does as well.

Of course, changing the basis results in the same map to $\Lambda(X, \mathbf{v}, \mathbf{w})$ via a different lift to the space of NQR's. Thus the map we have constructed is unique.

3.3. Connections with Kac-Moody algebras. Now assume that $X$ is simply laced (the corresponding Cartan matrix entries are all in $\{2,0,-1\})$, and let $\mathfrak{g}$ be the Kac-Moody algebra associated to the graph $X$. The geometry of the quiver variety $\Lambda$ interacts with the representation theory of $\mathfrak{g}$ in a number of interesting ways. For us, the important one will be the quiver model for the crystal $B_{\mu}$ described in Sav04.

Choose an orientation on $X$ with no oriented loops (for example, pick an ordering on the vertices, and direct the edges in ascending order). Let $\mu$ be a weight for $X$ with $\omega(\mu)=\mathbf{w}$, (i.e. $\left.\mu\left(\alpha_{i}^{\vee}\right)=w_{i}\right)$. Then we have the following remarkable result from Sai02

Theorem 3.2. (Saito) There is a natural $\mathfrak{g}$-crystal structure $C_{\mathbf{w}}$ on the components of

$$
\Lambda(X, \mathbf{w})=\bigsqcup_{\mathbf{v} \in \mathbb{Z}_{\geq 0}^{v(X)}} \Lambda(X, \mathbf{v}, \mathbf{w})
$$

such that

(1) $C_{\mathbf{w}} \cong B_{\mu}$.

(2) The weight map wt: $C_{\mathbf{w}} \rightarrow P(X)$ is given by

$$
\mathrm{wt}(c)=\mu-\alpha^{-1}(\mathbf{v})
$$

where $c \subset \Lambda(X, \mathbf{v}, \mathbf{w})$.

(3) The set of representations $\left\{(V, W, x, t) \mid \epsilon_{i}(x, t) \leq n\right\}$ is dense in a component $c$ if and only if $\tilde{e}_{i}^{n+1} c=0$, where, as before, $\tilde{e}_{i}$ is a Kashiwara operator.

Thus, the statistics of elements of the crystal which are important to us in determining tensor product multiplicities and branching rules have clear interpretations in terms of the associated quivers.

Consider the open subsets of $\Lambda(X)$ defined by

$$
\begin{aligned}
\Lambda_{\theta}^{\mu}\left(X, X_{0}\right) & =\left\{(V, W, x, t) \in \Lambda(X, \omega(\mu), \alpha(\mu-\theta)) \mid \epsilon_{i}(x, t)=0 \text { for all } i \in X_{0}\right\} \\
\Lambda_{\mu, \nu}^{\lambda}(X) & =\{(V, W, x, t) \in \Lambda(X, \omega(\mu), \alpha(\mu+\nu-\lambda)) \mid \epsilon(x, t) \leq \omega(\nu)\} .
\end{aligned}
$$


These can be interpreted as moduli spaces of NQR's with fixed representation theoretic properties.

As an immediate consequence of Theorem 3.2, we see the following proposition.

Proposition 3.3. For all weights $\lambda, \mu, \nu, \theta$,

- The branching multiplicity $b_{\theta}^{\mu}\left(X, X_{0}\right)$ is the number of components of $\Lambda_{\theta}^{\mu}\left(X, X_{0}\right)$.

- The tensor product multiplicity $c_{\mu, \nu}^{\lambda}$ is the number of components of $\Lambda_{\mu, \nu}^{\lambda}(X)$.

As was discussed in Section 2. we will also use the notation $\Lambda_{\mathbf{v}}^{\mathbf{w}}$ and $\Lambda_{\mathbf{w}, \mathbf{y}}^{\mathbf{v}}$, where $\mathbf{w}=\omega(\mu), \mathbf{y}=\omega(\nu)$ and $\mathbf{v}=\alpha(\mu+\nu-\lambda)=\alpha(\mu-\theta)$, interchangeably with $\Lambda_{\theta}^{\mu}$ and $\Lambda_{\mu, \nu}^{\lambda}$.

If $X$ is not simply laced, but symmetrizable, then we let $\Lambda(X, \mathbf{v}, \mathbf{w})$ be the appropriate closed subvariety of $\Lambda(\tilde{X}, \tilde{\mathbf{v}}, \tilde{\mathbf{w}})$, where $\tilde{X}$ is a simply laced diagram with an automorphism $a$ such that $\tilde{X} / a \cong X$ (see Sav04 for the details of this construction for arbitrary symmetrizable algebras). This corresponds to the well-known embedding of $B_{\mu}$ into the corresponding crystal graph for $\tilde{X}$.

\section{Stabilization OF QUiver VARIETIES}

4.1. Notation. Throughout this section, we fix weights $\mu, \nu, \lambda$, with $\mu+\nu-\lambda \in R(X)$. We let $\mathbf{w}=\omega(\mu), \mathbf{y}=\omega(\nu), \mathbf{v}=\alpha(\mu+\nu-\lambda)$, and $\lambda=\nu+\theta$.

Let $X$ and $X_{0}$ be as in the introduction, and assume that $\mathbf{w}$ and $\mathbf{y}$ vanish on $X_{0}$. From the definition, it follows that $\Lambda_{\mathbf{w}, \mathbf{y}}^{\mathbf{v}}(X) \subset \Lambda_{\mathbf{v}}^{\mathbf{w}}\left(X, X_{0}\right)$.

Fix an integer $s$, and let $X_{0}^{s}$ be the set of vertices in $X_{0}$ which are more than $s$ edges from the border of $X_{0}$, by which we mean the set of vertices in $X_{0}$ which are adjacent to edges in $X \backslash X_{0}$. For the remainder of this section, we assume that $X_{0}^{s+1}$ is non-empty.

Fix a vertex $i \in \mathcal{V}\left(X_{0}^{s+1}\right)$. By renumbering, we may assume that $i \in \mathcal{V}\left(X_{1}\right)$. Let $X^{\prime}$ be a graph with vertex set $(\mathcal{V}(X) \backslash\{i\}) \cup\left\{i^{\prime}, i^{\prime \prime}\right\}$ and edge set $\mathcal{E}(X) \cup\{d\}$, with one edge connected to $i$ attaching to $i^{\prime}$ and the other to $i^{\prime \prime}$ and $d$ connecting $i^{\prime}$ with $i^{\prime \prime}$.

We let $X_{0}^{\prime}$ be the obvious subgraph of $X^{\prime}$ which is also a disjoint union of type $A$ diagrams. This addition of an edge is shown in Figure 3 .

Now assume $\mathbf{v}$ is deep for $X_{0}^{s}$. It extends to a deep function $\mathbf{v}^{\prime}$ on $X^{\prime}$ in an obvious, unique way; outside of $X_{0}^{\prime s}$, it is defined by the graph isomorphism $X-X_{0}^{s} \cong X^{\prime}-X_{0}^{\prime s}$, and on $X_{0}^{\prime s}$ it simply takes the value of the 1-depth $\sigma_{1}$ on $i^{\prime}$ and $i^{\prime \prime}$, as is necessary to preserve deepness. We 
can extend $\mathbf{w}$ and $\mathbf{y}$ to $\mathbf{w}^{\prime}$ and $\mathbf{y}^{\prime}$ by the same process, since they are also deep for $X_{0}^{s+1}$.

4.2. Stabilization. As before, $X$ is the Dynkin graph of a symmetrizable Kac-Moody algebra, and $X_{0} \subset X$ is an elastic subgraph.

Theorem 4.1. Assume $v_{i} \leq s$ for all $i \in \mathcal{V}\left(X_{0}\right)$. If $\mathbf{v}$ is deep for $X_{0}^{s}$, there are canonical isomorphisms, preserving $\epsilon_{j}$ for all $j \in \mathcal{V}(X)$, such that

$$
\Lambda_{\mathbf{v}}^{\mathbf{w}}\left(X, X_{0}\right) \cong \Lambda_{\mathbf{v}^{\prime}}^{\mathbf{w}^{\prime}}\left(X^{\prime}, X_{0}^{\prime}\right)
$$

These in turn induce isomorphisms

$$
\Lambda_{\mathbf{w}, \mathbf{y}}^{\mathbf{v}}(X) \cong \Lambda_{\mathbf{w}^{\prime}, \mathbf{y}^{\prime}}^{\mathbf{v}^{\prime}}\left(X^{\prime}\right)
$$

If $\mathbf{v}$ is not deep for $X_{0}^{s}$, then $\Lambda_{\mathbf{v}}^{\mathbf{w}}\left(X, X_{0}\right)$, and hence $\Lambda_{\mathbf{w}, \mathbf{y}}^{\mathbf{v}}(X)$, is empty.

Corollary 4.2. If $b_{\theta}^{\mu}\left(X, X_{0}\right) \neq 0\left(\operatorname{resp} . c_{\mu, \nu}^{\lambda}(X) \neq 0\right)$ and $\alpha(\mu-\theta) \leq s$ on $X_{0}$, then $\mu-\theta$ is deep for $X_{0}^{s+1}$.

Corollary 4.3. $b_{\mathbf{v}}^{\mathbf{w}}\left(X, X_{0}\right)=b_{\mathbf{v}^{\prime}}^{\mathbf{w}^{\prime}}\left(X^{\prime}, X_{0}^{\prime}\right)$.

Corollary 4.4. $c_{\mathbf{w}, \mathbf{y}}^{\mathbf{v}}(X)=c_{\mathbf{w}^{\prime}, \mathbf{y}^{\prime}}^{\mathbf{v}^{\prime}}\left(X^{\prime}\right)$.

Applying these corollaries inductively, we can lengthen the strings in $X_{0}$ arbitrarily without affecting branching or tensor product multiplicities once $X_{j}(\mathbf{m})$ has at least $2 \sigma_{j}+1$ edges. Thus, Corollaries 4.3 and 4.4 imply part (1) of Theorem 1.3.

These corollaries seem to be stronger than the statement of Theorem [1.3, since in this case $\lambda$ is not assumed to vanish on $X_{0}$ but on $X_{0}^{s}$. But in fact, as Corollary 4.2 shows, $\lambda$ must vanish on $X_{0}^{s}$, and we lose nothing in the large $m$ limit by shrinking $X_{0}$ by a fixed number of vertices, so Theorem 1.3 is simply the asymptotic statement of Corollaries 4.3 and 4.4

Proof of Theorem 4.1. The idea of the proof is as follows: We show that on $X_{0}^{s}$, any stable quiver representation must have a very simple form, so there is a canonical way to extend it to an extra edge, or to remove an edge.

First, we assume that $X$ is simply-laced.

We must define some notation. Let $d_{i}$ and $d_{i+1}$ be the edges adjacent to $i$, and let $i+1$ be the vertex at the opposite end of $d_{i+1}$ (similarly for $i-1, i \pm n$, etc.). In $X_{0}^{\prime}$, we let $i^{\prime}$ be adjacent to $i-1$ and $i^{\prime \prime}$ to $i+1$.

For a quiver representation $(x, t)$ on $X$, we denote by $x_{d_{i}}$ the quiver map $V_{i} \rightarrow V_{i-1}$, and by $y_{d_{i}}=x_{\bar{d}_{i}}$ the map from $V_{i-1} \rightarrow V_{i}$. In the 


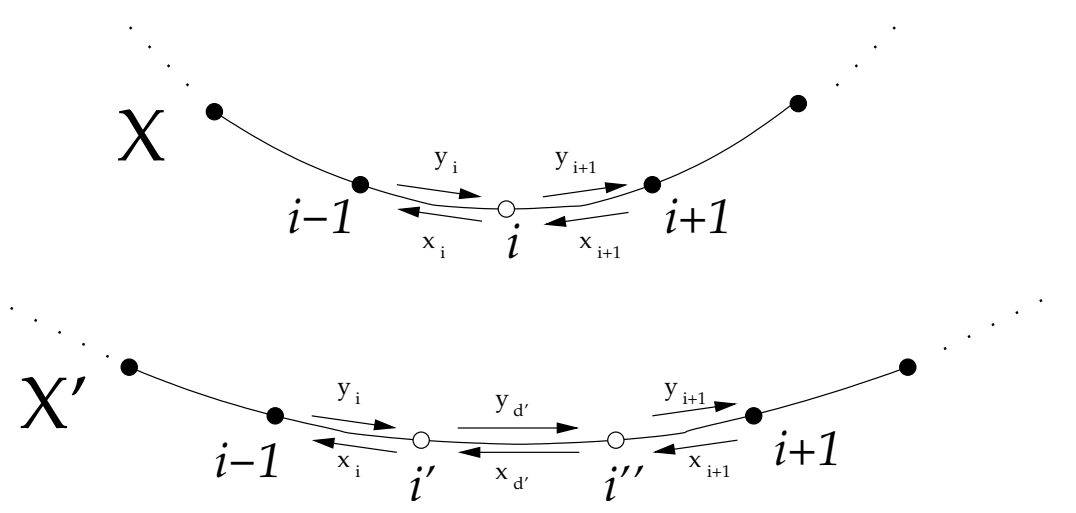

Figure 3 . The addition of an edge

notation of Section 3. this corresponds to choosing an orientation of $X_{0}$ with directed edges pointing in the decreasing direction.

We use $x$ and $y$ instead of just $x$ in order to simplify notation in the proof. When the point of application is obvious, we will drop the subscript from the maps $x_{d_{i}}$ and $y_{d_{i}}$. The notation defined above is shown in Figure 3 ,

If $(x, y) \in \Lambda(X, \mathbf{v}, \mathbf{w})$, it must satisfy the moment map condition, which reduces in the bivalent case to $y_{d_{i}} x_{d_{i}}=x_{d_{i+1}} y_{d_{i+1}}$. Now, apply this relation inductively to obtain the equalities

$$
y^{s} x^{s}=\left(y_{d_{i}} x_{d_{i}}\right)^{s}=x^{s} y^{s}
$$

By assumption, $y_{d_{i}} x_{d_{i}}: V_{i} \rightarrow V_{i}$ is nilpotent. Since $v_{i} \leq s,\left(y_{d_{i}} x_{d_{i}}\right)^{s}=$ 0 , and by (2), $x^{s} y^{s}=y^{s} x^{s}=0$ as well.

On the other hand, we claim:

$(*)\left(\operatorname{ker} x^{s}\right)_{j} \cap\left(\operatorname{ker} y^{s}\right)_{j}=0$.

If $z \in\left(\operatorname{ker} y^{s}\right)_{j} \cap\left(\operatorname{ker} x^{s}\right)_{j}$ and $z \neq 0$, then for some $\ell<s, y^{\ell} z \neq 0$, but $y^{\ell+1} z=0$. By assumption,

$$
x^{s} y^{\ell} z=y^{\ell} x^{s} z=0,
$$

so there exists an integer $k$, such that $z^{\prime}=x^{k} y^{\ell} z \neq 0$, but $x z^{\prime}=0$ and

$$
y z^{\prime}=x^{k}\left(y^{\ell+1} z\right)=0 .
$$

This contradicts stability, so the claim $(*)$ is true.

We claim also that for all $j \in \mathcal{V}\left(X_{0}\right)^{s}$ :

$$
(* *) V_{j}=\left(\operatorname{im} x^{s}\right)_{j}+\left(\operatorname{im} y^{s}\right)_{j} \text {. }
$$

We prove this by induction on $s$. For $s=1$, this is simply the condition $\epsilon_{j}((x, y), t)=0$. 
Assuming the claim for $k-1$, and applying the relation

$$
\left(\operatorname{im} x^{\ell_{1}} y^{\ell_{2}}\right)_{j}=\left(\operatorname{im} x^{\ell_{1}+1} y^{\ell_{2}}\right)_{j}+\left(\operatorname{im} x^{\ell_{1}} y^{\ell_{2}+1}\right)_{j}
$$

several times, we see that

$$
\begin{aligned}
V_{j}= & \left(\operatorname{im} x^{k-1}\right)_{j}+\left(\operatorname{im} y^{k-1}\right)_{j} \\
= & \left(\operatorname{im} x^{k}\right)_{j}+\left(\operatorname{im} y^{k}\right)_{j}+\left(\operatorname{im} x^{k-1} y\right)_{j}+\left(\operatorname{im} x y^{k-1}\right)_{j} \\
= & \left(\operatorname{im} x^{k}\right)_{j}+\left(\operatorname{im} y^{k}\right)_{j}+\left(\operatorname{im} x^{k-1} y^{2}\right)_{j}+\left(\operatorname{im} x^{2} y^{k-1}\right)_{j} \\
& \quad \vdots \\
= & \left(\operatorname{im} x^{k}\right)_{j}+\left(\operatorname{im} y^{k}\right)_{j}+\left(\operatorname{im} x^{k-1} y^{k}\right)_{j}+\left(\operatorname{im} x^{k} y^{k-1}\right)_{j} \\
= & \left(\operatorname{im} x^{k}\right)_{j}+\left(\operatorname{im} y^{k}\right)_{j}
\end{aligned}
$$

This proves the claim $(* *)$.

Since $x^{s} y^{s}=0,\left(\operatorname{im} x^{s}\right)_{j} \subseteq\left(\operatorname{ker} y^{s}\right)_{j}$ and $\left(\operatorname{im} y^{s}\right)_{j} \subseteq\left(\operatorname{ker} x^{s}\right)_{j}$. Applying $(*)$ and $(* *)$, we see that $V_{i}=\left(\operatorname{im} x^{s}\right)_{i} \oplus\left(\operatorname{im} y^{s}\right)_{i}=\left(\operatorname{ker} y^{s}\right)_{i} \oplus$ $\left(\operatorname{ker} x^{s}\right)_{i}$.

In particular, if $i-1$ is in $\mathcal{V}\left(X_{0}\right)^{s}, x_{d_{i}}$ is an isomorphism between $\left(\operatorname{im} x^{s}\right)_{i} \subset V_{i}$ and $\left(\operatorname{im} x^{s}\right)_{i-1} \subset V_{i-1}$ and $y_{d_{i}}$ an isomorphism between $\left(\operatorname{im} y^{s}\right)_{i-1} \subset V_{i-1}$ and $\left(\operatorname{im} y^{s}\right)_{i} \subset V_{i}$, and $v_{i}=v_{i-1}$. Applying this to all vertices in $X_{0}^{s}$, we see that if there is a stable quiver in $\Lambda(X, \mathbf{v}, \mathbf{w})$, then $v_{i}$ is constant on connected components of this set, implying the second part of our theorem.

This shows that on $X_{0}^{s}$, our quiver representation has a very simple structure. It naturally decomposes into two subrepresentations, one on which $x$ is an isomorphism, and one on which $y$ is an isomorphism, and the moment map condition guarantees that our representation will resemble Figure 4. This figure should be read such that every solid dot represents a basis vector of $V_{i}$, with leftward arrows representing the action of $x$ and the rightward $y$. Compare these to the case of $A_{\ell}$, covered by Frenkel and Savage in FS03, Sav03

From this picture, it is clear how to extend the pattern to the additional edge $d$. To be precise, we let $\beta^{\prime}: V_{i^{\prime}} \rightarrow V_{i}$ be an isomorphism, and similarly for $\beta^{\prime \prime}$. The map $x_{d}: V_{i^{\prime \prime}} \rightarrow V_{i^{\prime}}$ is defined by $\left(\beta^{\prime}\right)^{-1} \beta^{\prime \prime}$ on $\left(\beta^{\prime \prime}\right)^{-1}\left(\operatorname{im} x^{s}\right)_{i}$ and $\left(\beta^{\prime}\right)^{-1} x_{i+1} y_{i+1} \beta^{\prime \prime}$ on $\left(\beta^{\prime \prime}\right)^{-1}\left(\operatorname{im} y^{s}\right)_{i}$, and the same for $y$, mutatis mutandis.

We had to make an arbitrary choice of $\beta^{\prime}$ and $\beta^{\prime \prime}$, but we obtain an isomorphic NQR for any choice. In terms of our moduli theoretic interpretation, this says that we have a natural NQR of vector bundles for $X^{\prime}$ on $\Lambda_{\mathbf{v}}^{\mathbf{w}}\left(X, X_{0}\right)$, and thus a natural map $\varphi_{X, X^{\prime}}: \Lambda_{\mathbf{v}}^{\mathbf{w}}\left(X, X_{0}\right) \rightarrow$ $\Lambda_{\mathbf{v}^{\prime}}^{\mathbf{w}^{\prime}}\left(X^{\prime}, X_{0}^{\prime}\right)$. 


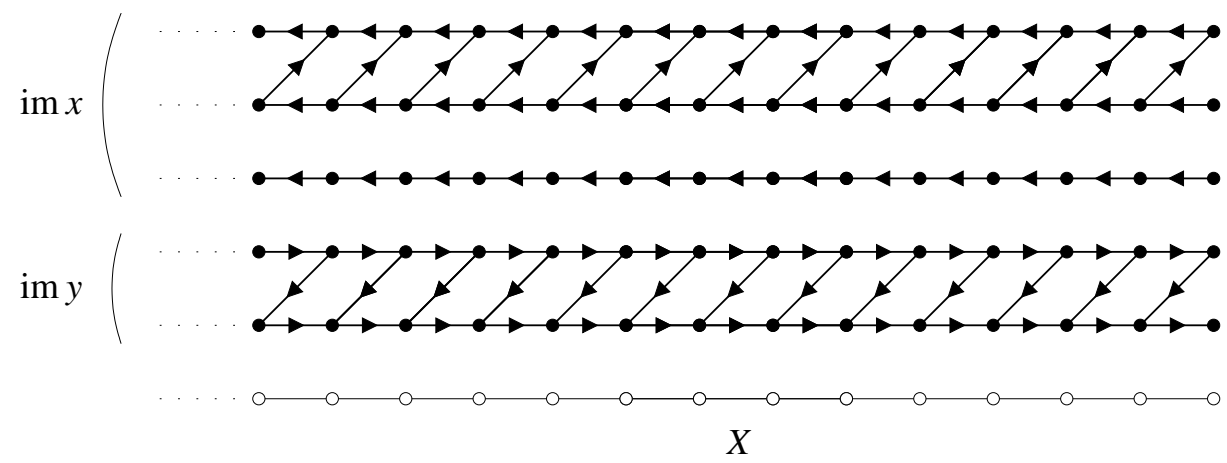

FiguRE 4. The structure of a quiver representation on $X_{0}^{s}$.

This map is in fact an isomorphism, since it has a clear inverse; given a quiver of the same form on $X^{\prime}$, the maps $x$ and $y$ are isomorphisms on $\left(\operatorname{im} x^{s}\right)_{i}$ and $\left(\operatorname{im} y^{s}\right)_{i}$, so we can just as easily perform the inverse operation of contracting an edge.

Note that we should not simply compose the quiver maps, as this will violate the the moment map condition, but instead must use $x$ and $y$ to identify the vector spaces on adjacent vertices.

Thus, we have a canonical bijection $\Lambda_{\mathbf{v}}^{\mathbf{w}}\left(X, X_{0}\right) \rightarrow \Lambda_{\mathbf{v}^{\prime}}^{\mathbf{w}^{\prime}}\left(X^{\prime}, X_{0}^{\prime}\right)$.

Any reader with concerns about whether this bijection is in fact an algebraic isomorphism, need only note that the process of adding or contracting an edge can be performed in families, so it gives an isomorphism between the functors representing $\Lambda_{\mathbf{v}}^{\mathbf{w}}\left(X, X_{0}\right)$ and $\Lambda_{\mathbf{v}^{\prime}}^{\mathbf{w}^{\prime}}\left(X^{\prime}, X_{0}^{\prime}\right)$. Since the scheme representing a functor is unique up to isomorphism, these varieties are canonically isomorphic.

This isomorphism preserves $\epsilon_{j}$ for all $j \in \mathcal{V}(X)$, since if $j$ is not in one of the $A_{n}$ strings, then the local structure of the quiver representation is unchanged. Thus it also induces an isomorphism

$$
\Lambda_{\mathrm{w}, \mathbf{y}}^{\mathbf{v}}(X) \cong \Lambda_{\mathbf{w}^{\prime}, \mathbf{y}^{\prime}}^{\mathbf{v}^{\prime}}\left(X^{\prime}\right) .
$$

If $X$ is not simply laced, we simultaneously apply the result to all vertices covering $i$ in the simply laced diagram $\tilde{X}$, and deduce the same result.

\section{The POLYNOMIAL BeHAVIOR OF WEIGHT MULTIPLICITIES}

As another consequence of our quiver theoretic picture of stabilization, we consider the behavior of weight multiplicities in the large $m$ limit of our sequence of diagrams. 
As with tensor product and branching rules, the dependence of weight multiplicities on the weights involved can be rewritten in terms of functions $\mathcal{V}(X) \rightarrow \mathbb{Z}$. We let $w_{\theta}^{\mu}=w_{\mathbf{v}}^{\mathbf{w}}$, using the notation of Section 2 ,

The behavior of weight multiplicities is considerably more subtle than that of branching rules. For example, the branching rule from $\mathfrak{s l}_{m}$ to the Levi component of a parabolic is simple, well-known, and stabilizes as $m \rightarrow \infty$, as is clear from their description in terms of tableaux, or partial Gelfand-Tsetlin patterns. On the other hand, the Weyl character formula is computationally quite unwieldy. However, the large $m$ asymptotics of weight multiplicities for $\mathfrak{s l}_{n}$ are reasonably well understood.

Theorem 5.1. ([BKLS99] Let $X \cong A_{\ell}$ and assume $\mathbf{w}$ vanishes on some connected subdiagram $X_{0}$ and $\mathbf{v}$ is of depth $s$ on $X_{0}$. Then for $m$ sufficiently large, $w_{\mathbf{v}}^{\mathbf{w}}(m)$ is equal to a polynomial in $m$ of degree $\leq s$.

Now, using the results of the previous section, we can reduce the general case to the above result for $A_{\ell}$.

As in the introduction, we can decompose $X_{0}(\mathbf{m})$ into its connected components

$$
X_{0}(\mathbf{m})=\bigsqcup_{i=1}^{k} X_{i}\left(m_{i}\right) \cong A_{\ell_{i}+m_{i}}
$$

and let $\sigma_{j}$ be the $j$-depth of $\mathbf{v}$.

Theorem 5.2. Let $X, X_{0}, \mathbf{w}, \mathbf{v}$ be as in Theorem 4.1. Then, for $\mathbf{m}$ sufficiently large, the weight multiplicity $w_{\mathbf{v}}^{\mathbf{w}}(\mathbf{m})$ is a polynomial in the variables $m_{1}, \ldots, m_{k}$ of multidegree less than $\boldsymbol{\sigma}=\left(\sigma_{1}, \ldots, \sigma_{k}\right)$.

This immediately implies Theorem 1.3. part (2).

Lemma 5.3. $w_{\theta}^{\mu}(X)=\sum_{\xi \in R^{+}\left(X_{0}\right)} b_{\theta+\xi}^{\mu}\left(X, X_{0}\right) \cdot w_{\theta}^{\theta+\xi}\left(X_{0}\right)$.

Proof. Let $\mathfrak{g}$ and $\mathfrak{g}_{0}$ be the Kac-Moody algebras corresponding to $X$ and $X_{0}$ respectively, and let $\mathfrak{t}$ be a Cartan subalgebra of $\mathfrak{g}$. Any integral weight $\mu$ of $X$ can be considered as a integral weight on the reductive Lie algebra $\mathfrak{g}_{0}+\mathfrak{t}$. Let $Z_{\mu}$ be the corresponding finite dimensional highest weight representation. As with any reductive group,

$$
V_{\mu} \cong \bigoplus_{\theta \in P(X)} \operatorname{Hom}_{\mathfrak{g}_{\mathfrak{o}}+\mathfrak{t}}\left(Z_{\lambda}, V_{\mu}\right) \otimes Z_{\lambda}
$$

The weight $\theta$ will only appear in the $\lambda$-isotypic component if $\lambda-\theta \in$ $R^{+}\left(X_{0}\right)$, the positive cone in the root lattice of $X_{0}$, so the $\theta$ weight 
space of $V_{\mu}$ can be decomposed as follows:

$$
\operatorname{Hom}_{\mathfrak{t}}\left(\mathbb{C}_{\theta}, V_{\mu}\right)=\bigoplus_{\xi \in R\left(X_{0}\right)} \operatorname{Hom}_{\mathfrak{t}}\left(\mathbb{C}_{\theta}, Z_{\theta+\xi}\right) \otimes \operatorname{Hom}_{\mathfrak{g}_{\mathfrak{o}}+\mathfrak{t}}\left(Z_{\theta+\xi}, V_{\mu}\right)
$$

Taking dimensions, the result follows.

Proof of Theorem 5.2. In our notation, we can rewrite Lemma 5.3 as:

$$
w_{\mathbf{v}}^{\mathbf{w}}(X(\mathbf{m}))=\sum_{\mathbf{z}: \mathcal{V}\left(X_{0}\right) \rightarrow \mathbb{Z}_{\geq 0}} b_{\mathbf{v}-\mathbf{z}}^{\mathbf{w}}(\mathbf{m}) \cdot w_{\mathbf{v}_{0}}^{\mathbf{v}_{0}+\mathbf{z}}\left(X_{0}(\mathbf{m})\right) .
$$

While the set $\mathbb{Z}_{>0}^{\mathcal{V}\left(X_{0}\right)}$ is infinite, the sum above is finite, since $b_{\mathbf{v}-\mathbf{z}}^{\mathbf{w}}(\mathbf{m})$ is non-zero for only finitely many $\mathbf{z}$.

Since $\mathbf{v}-\mathbf{z} \leq \mathbf{v}$, and $\mathbf{v}$ is of depth $s$, we can apply Corollary 4.2 to see that if $b_{\mathbf{v}-\mathbf{z}}^{\mathbf{w}}\left(X, X_{0}\right) \neq 0$, then $\mathbf{v}-\mathbf{z}$ is deep for $X_{0}^{s}$ of depth $\leq s$.

The set of weights deep on $X_{0}^{s}$ of depth $\leq s$ is independent of $m$ for large $m$, as is $b_{\mathbf{v}-\mathbf{z}}^{\mathbf{w}}\left(X, X_{0}\right)$. Thus, the set of $\mathbf{z}$ with $b_{\mathbf{v}-\mathbf{z}}^{\mathbf{w}}\left(X, X_{0}\right) \neq 0$ stabilizes as $\mathbf{m} \rightarrow \infty$.

Thus, for large values of $\mathbf{m}$, the sum in (3) is over a fixed set. Its summands are polynomial since $b_{\mathbf{v}-\mathbf{z}}^{\mathbf{w}}(\mathbf{m})$ stabilizes for all $y$, and by Theorem 5.1, the weight multiplicity $w_{\mathbf{v}_{0}}^{\mathbf{v}_{0}+\mathbf{z}}\left(X_{0}(\mathbf{m})\right)$ is a polynomial of multidegree less than $\boldsymbol{\sigma}$. Thus, $w_{\mathbf{v}}^{\mathbf{w}}(\mathbf{m})$ is also a polynomial of multidegree $\leq \boldsymbol{\sigma}$ in the variables $m_{1}, \ldots, m_{n}$.

\section{REFERENCES}

[BBL90] G. M. Benkart, D. J. Britten, and F. W. Lemire. Stability in modules for classical Lie algebras - a constructive approach. Mem. Amer. Math. Soc., 85(430):vi+165, 1990.

[BKLS99] Georgia Benkart, Seok-Jin Kang, Hyeonmi Lee, and Dong-Uy Shin. The polynomial behavior of weight multiplicities for classical simple Lie algebras and classical affine Kac-Moody algebras. In Recent developments in quantum affine algebras and related topics (Raleigh, NC, 1998), volume 248 of Contemp. Math., pages 1-29. Amer. Math. Soc., Providence, RI, 1999.

[Bry89] Ranee Kathryn Brylinski. Stable calculus of the mixed tensor character. I. In Séminaire d'Algèbre Paul Dubreil et Marie-Paul Malliavin, 39ème Année (Paris, 1987/1988), volume 1404 of Lecture Notes in Math., pages 35-94. Springer, Berlin, 1989.

[CP95] Vyjayanthi Chari and Andrew Pressley. A guide to quantum groups. Cambridge University Press, Cambridge, 1995. Corrected reprint of the 1994 original.

[FS03] Igor B. Frenkel and Alistair Savage. Bases of representations of type $A$ affine Lie algebras via quiver varieties and statistical mechanics. Int. Math. Res. Not., (28):1521-1547, 2003.

[Han85] P. Hanlon. On the decomposition of the tensor algebra of the classical Lie algebras. Adv. in Math., 56(3):238-282, 1985. 
[KS97] Masaki Kashiwara and Yoshihisa Saito. Geometric construction of crystal bases. Duke Math. J., 89(1):9-36, 1997.

[KV04] Michael Kleber and Sankaran Viswanath. Tensor product stabilization in Kac-Moody algebras, Advances in Mathematics, 201: 1-35, 2006.

[Lus91] G. Lusztig. Quivers, perverse sheaves, and quantized enveloping algebras. J. Amer. Math. Soc., 4(2):365-421, 1991.

[Nak94] Hiraku Nakajima. Instantons on ALE spaces, quiver varieties, and KacMoody algebras. Duke Math. J., 76(2):365-416, 1994.

[Nak98] Hiraku Nakajima. Quiver varieties and Kac-Moody algebras. Duke Math. J., 91(3):515-560, 1998.

[Pro] Nicholas J. Proudfoot. Hyperkahler analogues of Kahler quotients. Ph. D. thesis, U.C. Berkeley, Spring 2004, arXiv:math.AG/0405233

[Sai02] Yoshihisa Saito. Crystal bases and quiver varieties. Math. Ann., 324(4):675-688, 2002.

[Sav03] Alistair Savage. Geometric and combinatorial realizations of crystal graphs, Algebr. Represent. Theory, 9(2):161-199, 2006.

[Sav04] Alistair Savage. A geometric construction of crystal graphs using quiver varieties: Extension to the non-simply laced case, Infinite-dimensional aspects of representation theory (Charlottesville, VA, 2004), 133-154, Contemp. Math., 392, Amer. Math. Soc., Providence, RI, 1999.

[Vis05] Sankaran Viswanath. Dynkin diagram sequences and stabilization phenomena, to appear in Communications in Algebra, arXiv:math.RT/0505616

Department of Mathematics, University of California, Berkeley, 970 Evans Hall, Berkeley, CA 94720

E-mail address: bwebste@math.berkeley.edu

$U R L:$ http://math.berkeley.edu/ bwebste 